\title{
Theoretical Study and Experimental Optimization on the Reliability of a Seawater Hydraulic Solenoid Valve
}

\author{
Shan Wu, Xufeng Zhao, Donglin Li, and Xiaohui Li
}

\begin{abstract}
As an important component of the variable ballast system (VBS), an key subsystem of submersible, seawater hydraulic solenoid shut-off valve(SSV) functions as a transport hub which controls the discharge and injecting of variable ballast cabin. Through theoretical analysis, temperature rise was found to be a main cause of the SSV's failure. In order to improve the reliability of the SSV, two experimental apparatus were built to test the characteristics of the solenoids and the reliability of the SSV. Result shows variable voltage driving is an effective way of reducing the failure, which can sharply restrain the temperature rise of the solenoids. Moreover, an optimal action cycle was found in which the solenoid valve has a longer continuous on-off time between failures (CTBF) and shorter response time.
\end{abstract}

Index Terms-Seawater hydraulics, variable ballast system, solenoid shut-off valve, failure analysis.

\section{INTRODUCTION}

Manned submersible is a very important equipment for deep-sea exploration, the main mission of which is carrying scientists, engineers, various instruments and tools to the deep sea to perform tasks of exploration, scientific research [1]. During one dive, the total weight of the submersible often varies because of the change of the load in the scientific basket or the difference of seawater density at different depths or regions. The VBS is designed for the pilot to adjust the weight of the submersible while in the deep sea [2].

Fig. 1 shows the schematic diagram of a typical variable ballast system [3]-[5]. In the system, the seawater solenoid valves manifold is composed of four solenoid valves and controls the discharge and influxion of water from and to ballast cabin [6], [7]. The appearance of the valve manifold is shown in Fig. 2.

The reliability of the valve manifold is particularly important as it plays a key role in VBS. In this paper, the failure model of a single valve in the valve manifold will be analyzed theoretically and experimentally. In addition, the measures to reduce failures are presented and verified.

Manuscript received December 11, 2014; revised March 25, 2015.This work was supported by 2014 Scientific Research Project of Hubei Provincial Department of Education (B2014168) and 2013 Scientific Research Project (Key Project) of Wuhan Technology and Business University (B2013005).

$\mathrm{S}$. Wu is with the Wuhan Technology and Business University, No. 3, Huangjiahu West Road, Wuhan 430065, China (e-mail: hariny@ 163.com).

X. F. Zhao and D. L. Li are with the School of the Mechanical Science and Engineering, Huazhong University of Science and Technology, No.1037, Luoyu Road, Wuhan 430074, China (e-mail: xufengzhao@vip.qq.com, lidonglin@hust.edu.cn).

X. H. Li is with the Shenzhen Research Institute of Xiamen University, A600-602, Virtual University Park, South Zone of High-tech, Shenzhen 518063, China (e-mail: lixiaohui@hust.edu.cn).

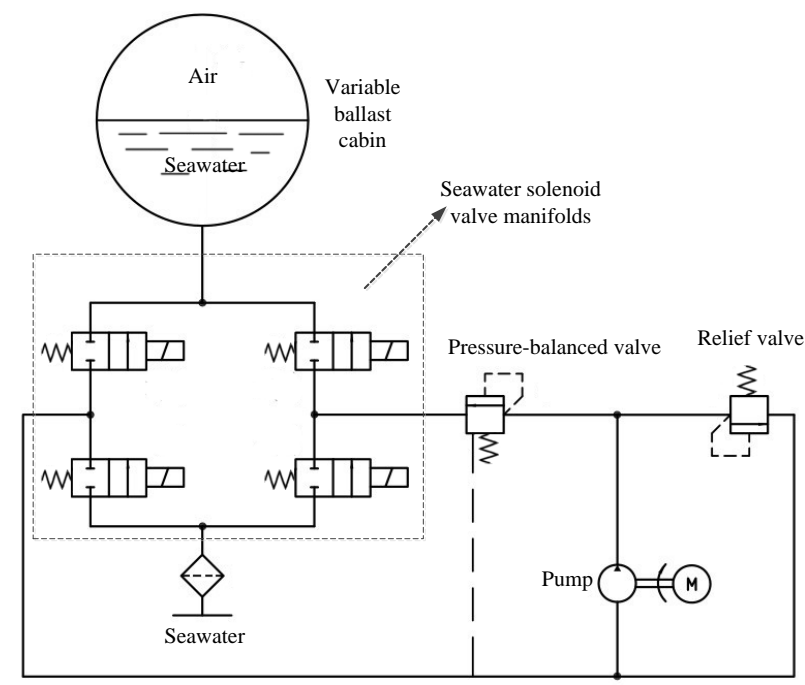

Fig. 1. Schematic diagram of variable ballast system.

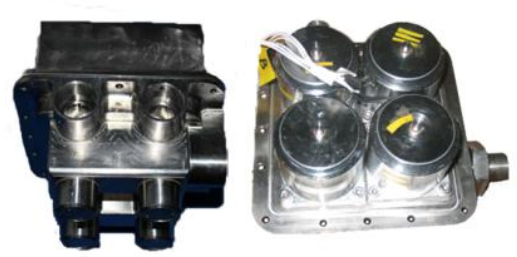

Fig. 2. The appearance of the valve manifold.

\section{THEORETICAL ANALYSIS OF FAILURES}

The failure of solenoid valve is mostly caused by temperature rise [8]. When temperature rises, the output force of solenoids will decrease and the frictions of solenoid valve will increase as a result of thermal expansion [9]. In order to learn more about the failures, the force of the valve poppet and temperature rise of the solenoid will be analyzed in this section.

\section{A. Force on the Valve Poppet}

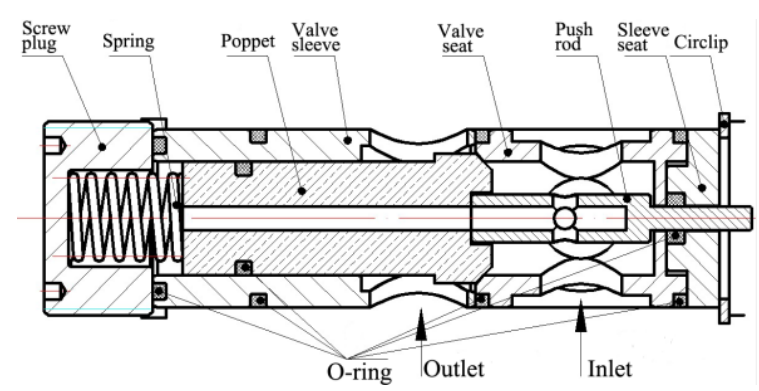

Fig. 3. Structure diagram of single solenoid valve.

Fig. 3 shows the structure of a single solenoid valve in the valve manifold [4]. The solenoid pushes valve poppet by means of the push rod which is shown in Fig. 3. 
According to Fig. 3, the force balance equation of the valve poppet can be:

$$
m a=F+f_{\text {in }}-f_{c}-f_{\text {out }}-f_{t} \pm f_{f}-f_{s}-f_{h} \pm G
$$

where, $m$ is the weight of the moving parts, $a$ is the acceleration of the moving parts, $F$ is the force exerted by solenoid, $f_{\text {in }}$ is the force on the poppet exerted by static pressure in the direction of inlet, $f_{c}$ is the force on the poppet exerted by static pressure in the spring chamber, $f_{\text {out }}$ is the force on the poppet exerted by static pressure in the direction of outlet, $f_{t}$ is the force on the push rod exerted by static pressure, $f_{f}$ is the total friction on the poppet, $f_{s}$ is the Bernoulli force, $f_{h}$ is force exerted by the spring, $\mathrm{G}$ is gravity of the moving parts.

Assuming that the initial force exerted by the spring is $f_{\mathrm{h} 0}$ and the solenoid is in good condition, the force on the poppet should meet the given equation as follows:

In the state of shut-off:

$$
f_{h 0}+G+f_{c}>f_{\text {in }}
$$

When solenoid is energized:

$$
F_{\text {min }}-f_{t}+f_{\text {in }}>f_{h 0}+G+f_{c}+\lambda f_{f}
$$

In the opening process:

$$
F-f_{t}+f_{i n}>f_{h}+G+f_{c}+f_{f}+f_{\text {smax }}
$$

In the state of fully open:

$$
F_{\text {max }}-f_{t}+f_{\text {in }}>f_{\text {hmax }}+G+f_{c}+f_{\text {smin }}
$$

When the solenoid losses power:

$$
f_{\text {hmax }}+G+f_{c}+f_{\text {smin }}>f_{\text {in }}+\lambda f_{f}
$$

In the closing process:

$$
f_{h}+G+f_{c}+f_{s}>f_{\text {in }}+f_{f}
$$

\section{B. Temperature Rise of Solenoid}

As the solenoid in SSV is a DC solenoid, the main cause of temperature rise is Joule heat [6]. The effect of Joule heat complies with Joule's law:

$$
Q=\left(U^{2} / R\right) t
$$

where $Q$ is Joule heat, $U$ is the energizing voltage, $R$ is the resistance of solenoid. The relationship between Joule heat and temperature rise is:

$$
P d t=C G d \tau+\mu s \tau d t
$$

where $P$ is the Joule heat of solenoid, $C G d \tau$ is the heat energy which rises temperature, $\mu s \tau d t$ is the heat energy of heat exchange..

\section{Cause of Failure}

According to Eq. (3)-Eq. (7), the increase of friction which is caused by thermal expansion leads to failures of opening operation and closing operation.

As temperature rises, the resistance of solenoid increases and the current through the solenoid decreases if the energizing voltage is kept constant. Since the electromagnetic force is proportional to ampere turns, the force exerted by solenoid decreases with temperature rising. Based on Eq. (3)-Eq. (5), the decrease of the force exerted by the solenoid leads to the failure of opening operation. The opening process of SSV is shown in Fig. 4. As the volume and the weight of SSV are limited, it is extravagant to select a larger solenoid which can completely meet the requirement in hot state. Therefore, it is important to find some improved measures to decrease the failure ratio of the volume-limited SSV.

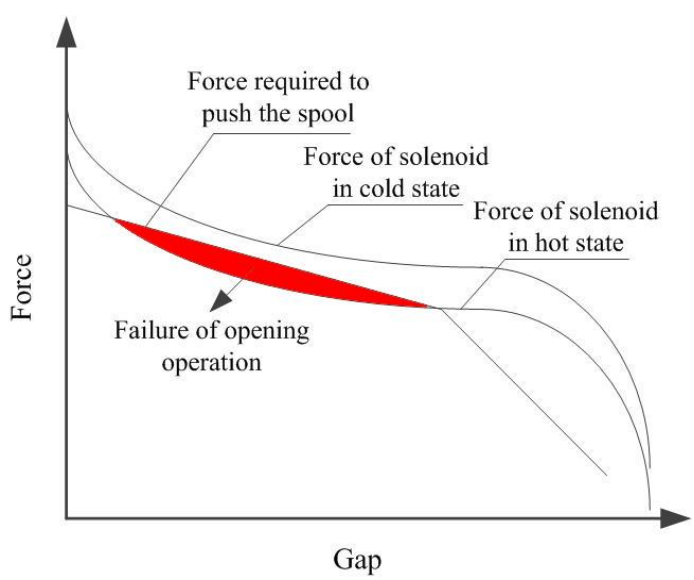

Fig. 4. Opening process of a single solenoid valve.

\section{EXPERIMENTAL APPARATUS}

In order to find some measures to improve the reliability of SSV, the experimental apparatus of solenoid and SSV were designed respectively.

\section{A. Experimental Apparatus of Solenoid}

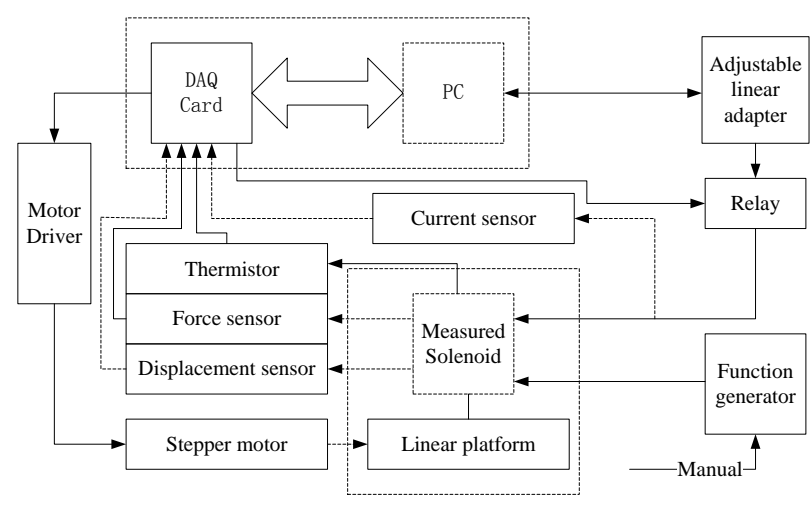

Fig. 5. System chart of solenoid experimental apparatus.

The system schematics of the experimental apparatus is shown in Fig. 5. The mechanical part of the apparatus consists of stepper motor, linear platform and mechanical bracket which are shown in Fig. 6. It supports all devices and can be used to adjust the length of solenoid's air gap according to the control signal. The control part consists of linear adapter, function generator, DAQ module, sensors, relay and measuring software. Measuring software collects 
sensor signals through DAQ module to decide the next experiment parameters and generates experimental reports as well.

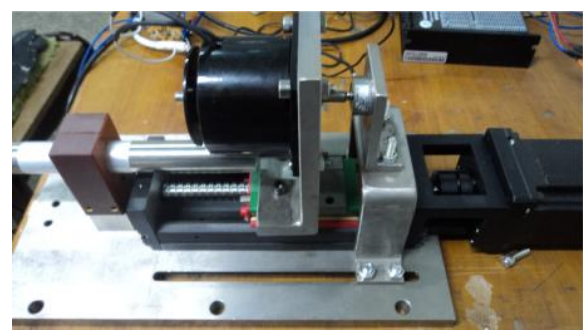

Fig. 6. Mechanical part of solenoid experimental apparatus

\section{B. Experimental Apparatus of SSV}

The hydraulic scheme of the experimental apparatus is shown in Fig. 7 which can set flow and pressure of the system by adjusting the throttle valve. In the experiment, experimental conditions were set to $2.4 \mathrm{MPa}$ and $25 \mathrm{~L} / \mathrm{min}$ which are the rated specifications of SSV. The measuring system controls the valve's on-off by USB-4711A DAQ card which connects a relay on the output port and diagnose the failures of SSV through the signals of a pressure sensor. If the pressure of the sensor is more than $0.2 \mathrm{MPa}$ in state of fully open, the valve is not fully opened and a failure of opening operation is figured. Similarly, if the pressure of sensor is less than $2.2 \mathrm{MPa}$ in state of shut-off, the valve is not fully closed and a failure of closing operation is figured. Experimental parameters such as action cycle, duty cycle, failure pressure and numbers of operations can be set in the programs.

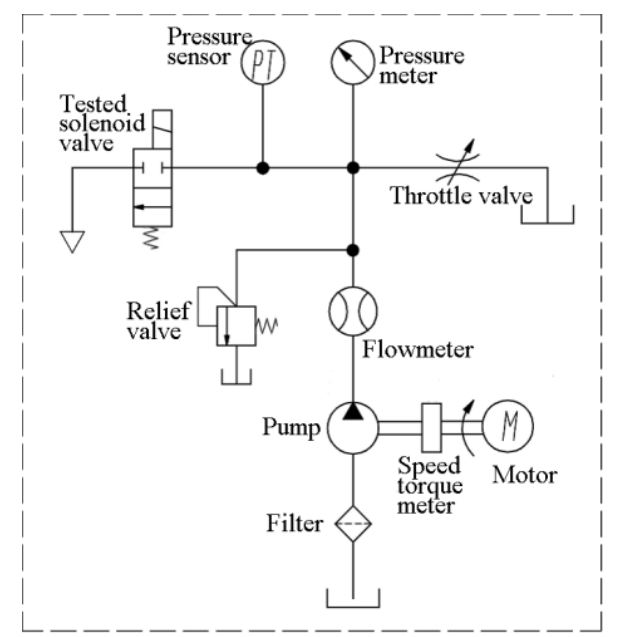

Fig. 7. Hydraulic scheme of valve experimental platform.

\section{EXPERIMENTAL RESULTS AND IMPROVEMENTS}

Fig. 8 shows the temperature of solenoid has increased by 2.8 times in a continuous duty state after $45 \mathrm{mins}$, and the output force of the solenoid decreased by $40 \%$. In fact, many existing measures of improving the reliability are based on restraining the temperature rise of solenoid. In the continuous duty state, variable voltage driving is a good way to improve reliability. Due to coupled response of valve and solenoid in the critical state, there is an optimal action cycle to ensure the rapidity and reliability of $\mathrm{SSV}$ in a continuous fast on-off state.

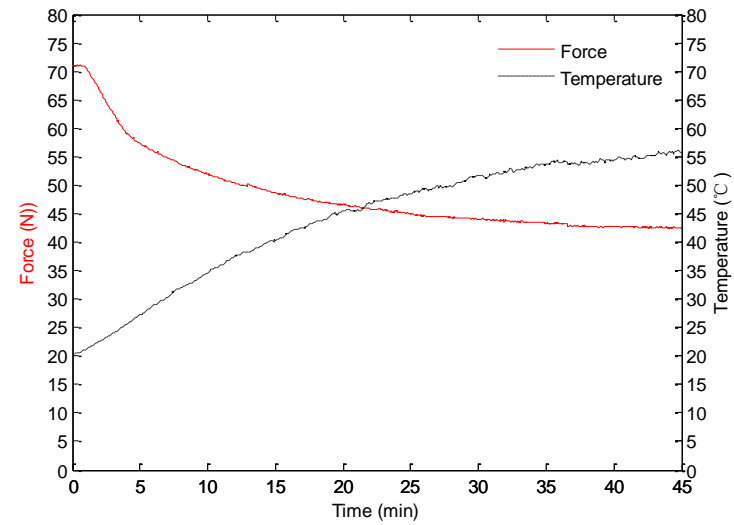

Fig. 8. Characteristics of a continuous energized solenoid.

The SI unit for magnetic field strength $H$ is $\mathrm{A} / \mathrm{m}$. However, if you wish to use units of $T$, either refer to magnetic flux density $B$ or magnetic field strength symbolized as $\mu_{0} H$. Use the center dot to separate compound units, e.g., "A $\cdot \mathrm{m}^{2}$."

\section{A. Variable Voltage Driving}

The force exerted by solenoid increases faster than the force required to push valve poppet in the opening process. Figure 9 illustrates the force required to push the valve poppet which is calculated by Eq. (2)-Eq. (7) and the force-displacement characteristics of the solenoid driven by different voltage which is measured at ambient temperature of $20{ }^{\circ} \mathrm{C}$.

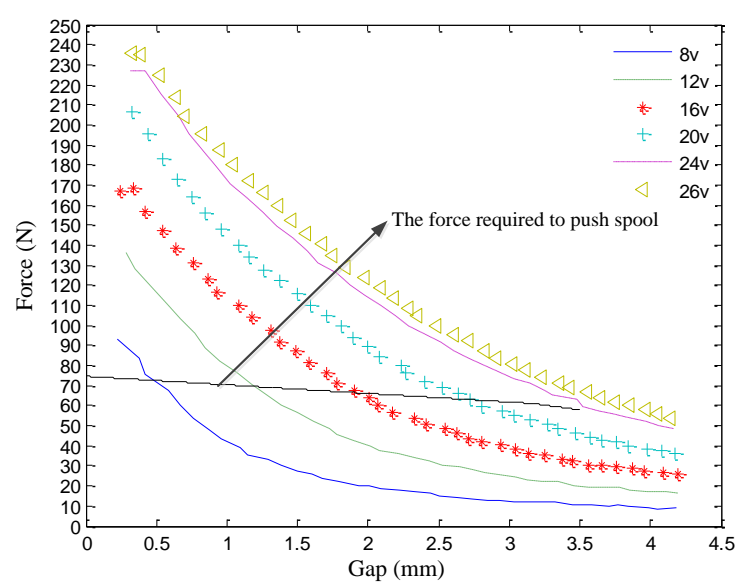

Fig. 9. Force of solenoid energized by different voltage.

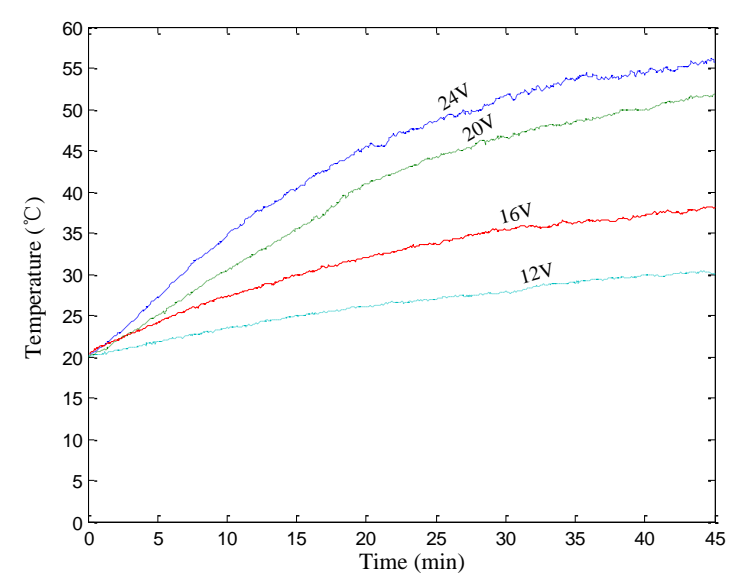

Fig. 10. Temperature rise of solenoid.

When the solenoid valve has been fully opened, it is inefficient to energize the solenoid by $24 \mathrm{~V}$ voltage, since 
$12 \mathrm{~V}$ energized voltage is able to provide sufficient force for maintaining the opening state. Higher energized voltage means more Joule heat which leads to temperature rise. Fig. 10 shows the temperature rise of a solenoid energized by different voltage for $45 \mathrm{~min}$ at ambient temperature of $20{ }^{\circ} \mathrm{C}$. The temperature rise of the solenoid energized by $24 \mathrm{~V}$ voltage is $260 \%$ higher than that of the one energized by $12 \mathrm{~V}$ voltage. Unquestionably, the way that opens the valve by a higher voltage and maintains the opening state by a lower voltage will effectively improve the reliability of SSV.

\section{B. Optimal Action Cycle}

Experimental results illustrating in Table I show that the continuous on-off times between failures (CTBF) of the solenoids operating with different action cycles and the same duty cycle are different from each other. The same duty cycle means the same size of Joule heat and the same cooling time. In theory, the temperature rise of the solenoid should be independent of action cycle under the same duty cycle. Experimental results in Table II which show the temperature rise of the solenoid energized by $24 \mathrm{~V}$ voltage support this point.

TABLE I: CTBF OF $50 \%$ DUTY CYCLE $\left(34^{\circ} \mathrm{C}\right)$

\begin{tabular}{ccc}
\hline \hline Action cycle & Number of operations & CTBF \\
\hline $2 \mathrm{~s}$ & 450 & $15 \mathrm{~min}$ \\
$4 \mathrm{~s}$ & 762 & $50 \mathrm{~min} 48 \mathrm{~s}$ \\
$5 \mathrm{~s}$ & 677 & $56 \mathrm{~min} 25 \mathrm{~s}$ \\
$6 \mathrm{~s}$ & 595 & $59 \mathrm{~min} 30 \mathrm{~s}$ \\
\hline \hline
\end{tabular}

The critical state, in which the force exerted by the solenoid is close to the force required to push the poppet, a little fluctuation of electromagnetic force or the required force may cause failure of opening operation. When the solenoid is energized, the current through the solenoid increases rapidly. The changing current brings inductive effect which causes brief drop of the current [10]. As the force exerted by solenoid is positive related to the current through solenoid, there is a concomitant brief drop in electromagnetic force when the SSV is opening. Additionally, in critical state, the transient flow force which is neglected in the design is large enough to frustrate the opening operation. The transient flow force and the brief drop in the force exerted by solenoid lead to the delay in the opening operation in critical state. There is an obvious interrupt in opening operation when the solenoid valve was tested in critical state. A series of experimental results show that $4 \mathrm{~s}$ is an optimal action cycle considering the rapidity and reliability simultaneously.

TABLE II: TEMPERATURE RISE OF 50\% DUTY CYCLE $\left(20^{\circ} \mathrm{C}\right)$

\begin{tabular}{cccc}
\hline \hline Action cycle & $10 \mathrm{~min}$ & $20 \mathrm{~min}$ & $30 \mathrm{~min}$ \\
\hline $2 \mathrm{~s}$ & $5.3^{\circ} \mathrm{C}$ & $9.2^{\circ} \mathrm{C}$ & $11.3^{\circ} \mathrm{C}$ \\
$4 \mathrm{~s}$ & $5.2^{\circ} \mathrm{C}$ & $9.4^{\circ} \mathrm{C}$ & $11.9^{\circ} \mathrm{C}$ \\
$6 \mathrm{~s}$ & $5.0^{\circ} \mathrm{C}$ & $9.4^{\circ} \mathrm{C}$ & $11.9^{\circ} \mathrm{C}$ \\
$10 \mathrm{~s}$ & $5.3^{\circ} \mathrm{C}$ & $9.4^{\circ} \mathrm{C}$ & $11.9^{\circ} \mathrm{C}$ \\
\hline \hline
\end{tabular}

\section{Improvements and Comparisons}

Based on series of experiments, it was found that the following measures can significantly improve the reliability of SSV:

1) Variable voltage driving: opens the valve by a higher voltage and maintains the opening state by a lower voltage;

2) Using the optimal action cycle;

3) Adding lubricating oil to the guiding sleeve of solenoid. Table III shows the CTBF of the SSV with and without improvements.

\begin{tabular}{ccc}
\multicolumn{3}{c}{ TABLE III: COMPARISONS OF CTBF } \\
\hline \hline Improvements & Number of Operations & CTBF \\
\hline without & 762 & $50.8 \mathrm{~min}$ \\
with & 3749 & $250 \mathrm{~min}$ \\
& & \\
\hline \hline
\end{tabular}

\section{CONCLUSION}

Theoretical analysis and experimental results in this paper suggested that the temperature rise of SSV causes failure. Experimental results show that variable voltage driving can substantially restrain the temperature rise of the solenoid. In critical state, the brief drop in electromagnetic force and the transient flow force are the causes of delay in opening operation. There is an optimal action cycle which satisfies the requirements of both rapidity and reliability. The measures presented in this paper have been proven to be effective in reducing failures. In further, the coupled response of the valve and the solenoid need to be analyzed in detail to improve the response speed of SSV in critical state. In addition, the design of solenoid will be revised to restrain temperature rise.

\section{ACKNOWLEDGMENT}

This research was supported by 2014 Scientific Research Project of Hubei Provincial Department of Education (B2014168) and 2013 Scientific Research Project (Key Project) of Wuhan Technology and Business University (B2013005).

\section{REFERENCES}

[1] Z. L. Qiu, J. X. Leng, J. P. Chen, and G. W. Tang, "Research of variable ballast system in deep-sea manned submersible," Chinese Hydraulics \& Penumatics, vol. 11, pp. 9-11, 2003.

[2] Z. L. Qiu, "Design and research on a variable ballast system for deep-sea manned submersibles," Journal of Marine Science and Application, vol. 7, pp. 256-260, 2008.

[3] Y. S. Liu, D. F. Wu, D. L. Li, X. F. Zhao, and X. F. Li, "Applications of seawater hydraulics in deep-sea equipment," Journal of Mechanical Engineering, vol. 50, pp. 28-35, 2014.

[4] Q. Y. Hu, J. Zhou, and Z. Zha, "Application of PSO-BP network algorithm in AUV depth control," Applied Mechanics and Materials, vol. 321-324, pp. 2025-2031, 2013.

[5] Y. S. Liu, D. F. Wu, D. L. Li, and X. F. Zhao, "Seawater hydraulic buoyancy adjusting system for large-depth submersible," Chinese Hydraulics \& Penumatics, vol. 10, pp. 1-10, 2014.

[6] J. L. Chen, Y. S. Liu, D. F. Wu, X. Y. Mao, and T. Jiang, "The development of a buoyancy adjustment system for submersibles," Chinese Hydraulics \& Penumatics, vol. 1, pp. 79-83, 2012.

[7] X. Mao, "Development of seawater solenoid shut-off valves," Master thesis, Huazhong University of Science and Technology, 2012.

[8] S. V. Angadi, R. L. Jackson, and S. Y. Choe, "Reliability and life study of hydraulic solenoid valve," Engineering Failure Analysis, Part 2: Experimental study, vol. 16, pp. 944-963, 2009.

[9] S. V. Angadi, R. L. Jackson, and S. Y. Choe, "Reliability and life study of hydraulic solenoid valve," Engineering Failure Analysis, Part 1: A multi-physics finite element model, vol. 16, pp. 874-887, 2009. 
[10] K. Harmer, G. W. Jewel, and D. Howe, "Transient performance of a short-stroke linear solenoid actuator," IEE Proc.-Electr, vol. 149, pp. 379-38, 2002.

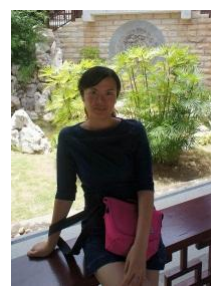

Shan Wu received the B.S. degree in applied mathematics from the Jianghan University, Wuhan, China, in 2001, and the M.S. degree from Huazhong University of Science and Technology in 2006. She is currently working toward the Ph.D. degree at Huazhong University of Science and Technology. In 2004, she joined the Wuhan Technology and Business University, where she has been an associate professor since 2011. Her current research interests include mathematical modeling and numerical simulation.

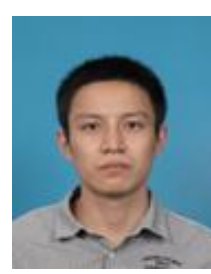

Xufeng Zhao received the B.S. degree from the Department of Control Science and Engineering, Huazhong University of Science and Technology, Wuhan, China, in 2007. He is currently working toward the Ph.D. degree at School of the Mechanical Science and Engineering, Huazhong University of Science and Technology. His current research interests include water hydraulic system and control, electro-hydraulic proportional control, and reciprocating process compressor.

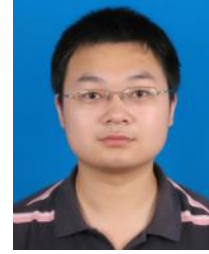

Donglin Li received the B.S. degree in mechanical design manufacturing and automation from China University of Geosciences, Wuhan, China, in 2011, $\mathrm{He}$ is currently working toward the Ph.D. degree at the School of Mechanical Science and Engineering, Huazhong University of Science and Technology. His current research interests include water hydraulic component research and development and water hydraulic transmission technology.

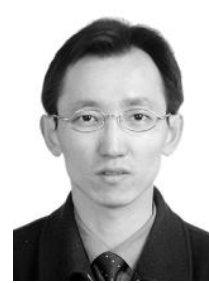

Xiaohui Li received the B.S. degree from the Department of Fluid Transmission and Control, Taiyuan Institute of Technology, Taiyuan, China, in 1994, and the M.S. and the Ph.D. degrees in mechanical engineering from Huazhong University of Science and Technology, in 1997 and 2012, respectively. In 1997, he joined the School of the Mechanical Science and Engineering, Huazhong University of Science and Technology, as an assistant professor. He is currently working as a research fellow of Shenzhen Research Institute of Xiamen University, Shenzhen, China. His current research interests include water jet propulsion and water mist technology. 